\title{
Induction of Humoral and Cellular Immunity by Intradermal Delivery of SARS-CoV-2 Nucleocapsid Protein Using Dissolvable Microneedles
}

\author{
Chaiyaporn Kuwentrai $\mathbb{D}^{1},{ }^{1}$ Jinming Yu $\mathbb{D}^{2},{ }^{2}$ Bao-zhong Zhang $\mathbb{D}^{3},{ }^{3}$ Ye-fan Hu $\mathbb{D}^{1,4}$ \\ Ying Dou $\left(\mathbb{\infty},{ }^{1}\right.$ Hua-rui Gong $\left({ }^{1},{ }^{1}\right.$ Jian-Dong Huang $\left({ }^{1},{ }^{1,3}\right.$ and Chenjie $\mathrm{Xu}\left(\oplus^{2}\right.$ \\ ${ }^{1}$ School of Biomedical Sciences, Li Ka Shing Faculty of Medicine, The University of Hong Kong, 3/F, Laboratory Block, \\ 21 Sassoon Road, Hong Kong SAR, China \\ ${ }^{2}$ Department of Biomedical Engineering, City University of Hong Kong, 83 Tat Chee Avenue, Kowloon, Hong Kong SAR, China \\ ${ }^{3}$ CAS Key Laboratory of Quantitative Engineering Biology, Shenzhen Institute of Synthetic Biology, Shenzhen Institutes of \\ Advanced Technology, Chinese Academy of Sciences, Shenzhen 518055, China \\ ${ }^{4}$ Department of Medicine, University of Hong Kong, 4/F Professional Block, Queen Mary Hospital, 102 Pokfulam Road, \\ Hong Kong SAR, China
}

Correspondence should be addressed to Jian-Dong Huang; jdhuang@hku.hk and Chenjie Xu; chenjie.xu@cityu.edu.hk

Received 26 January 2021; Accepted 4 May 2021; Published 18 May 2021

Academic Editor: Cinzia Milito

Copyright ( 92021 Chaiyaporn Kuwentrai et al. This is an open access article distributed under the Creative Commons Attribution License, which permits unrestricted use, distribution, and reproduction in any medium, provided the original work is properly cited.

\begin{abstract}
The nucleocapsid protein (NP) of the severe acute respiratory syndrome coronavirus 2 (SARS-CoV-2) contains immunogenic epitopes that can induce cytotoxic T lymphocyte (CTL) against viral infection. This makes the nucleocapsid protein a suitable candidate for developing a vaccine against SARS-CoV-2 infection. This article reports the intradermal delivery of NP antigen using dissolvable microneedle skin patches that could induce both significant $\mathrm{B}$ cell and $\mathrm{T}$ cell responses.
\end{abstract}

\section{Introduction}

The severe acute respiratory syndrome coronavirus 2 (SARS$\mathrm{CoV}-2)$ has triggered a global pandemic with extremely rapid infection [1-3]. Candidate vaccines are being tested for their ability to prevent infection/reinfection of SARS-CoV-2. Currently, there are several promising spike-based vaccines from different developers such as Pfizer/BioNTech and Sinovac, but these vaccines have yet achieved sterilizing immunity in the population. Furthermore, there is limited information on how long the protective immunity could last, especially when variants of SARS-CoV-2 are emerging constantly. Single-antigen vaccines may easily lose their protection effect due to the mutations. Therefore, our group aims to investigate nonspike vaccine candidates that may induce additional, long-lasting or more complete protective responses in patients. Apart from the conventional SARS-CoV-2 spike glycoprotein (S protein), the nucleocapsid protein (NP) has been identified as an alternative candidate for vaccine development due to its ability to induce antiviral cytotoxic $\mathrm{T}$ cell responses [4]. NP plays a vital role in viral host cell entry and modulates virus particle assemble and release [5]. Notably, there are many human leukocyte antigen (HLA) binding peptides within NP, which could prime specific CD8+ and $\mathrm{CD} 4+\mathrm{T}$ cell responses via major histocompatibility complex class 1 (MHC1) and major histocompatibility complex class 2 (MHC2) pathways, respectively [6]. Furthermore, immunoblot assays have revealed the presence of IgA, IgM, and IgG antibodies against $\mathrm{N}$ antigen in COVID-19 infection patients' sera [7]. More recently, Chukwudozie et al. developed a subcomponent vaccine targeting the SARS-CoV-2 nucleocapsid phosphoprotein RNA binding domain. This vaccine contains both antigenic $B$ cell and $T$ cell epitopes that provide a sufficient antigenic index and nonallergenic 
property [8]. Importantly, Peng et al. revealed that CD8+ T cell responses were associated with milder disease (2-3 weeks recovery rates), whereas more severe cases of SARS-CoV-2 had lower CD8+ T cell responses. Typically, it only takes 7 to 10 days for COVID-19 virus infections to activate antiviral $\mathrm{T}$ cell responses which correlate well with the time taken for milder disease patients to recover. This indicates that $\mathrm{T}$ cell immune responses play an important role in the prevention of SARs-COV-2 pathogenesis and possibly longer-term protective immunity [9]. The pathogenesis of SARS-CoV-2 involves impaired dendritic cell responses along with delayed $\mathrm{T}$ cell responses. These delayed $\mathrm{T}$ cell responses exhibited greater CD4+ T cells than CD8+ T cells [10]. Collectively, these preliminary evidences support the prospect of developing alternative nonspike vaccine candidates, such as NP, that could elicit specific $\mathrm{T}$ cell responses against SARS-CoV-2. Our group aims to investigate microneedle-delivered NP vaccines that may induce additional, long-lasting or more complete protective responses in patients.

The storage, transport, and administration of the SARSCoV-2 vaccine are vital considerations for vaccine development and control of the pandemic worldwide. The majority of approved SARS-CoV-2 vaccines, such as the PfizerBioNTech BNT162b2 vaccine or Moderna's mRNA-1273 vaccine, are administered by intramuscular (IM) injection. However, such conventional injection techniques may induce the risk of blood-related infections and require trained personnel to perform injections [11-15]. These constraints may be solved by other nonconventional techniques.

Microneedle- (MN-) based intradermal delivery offers unique benefits, such as reduced biohazard waste, stable storage at room temperature, ease of distribution, and pain-free vaccination. The rationale behind $\mathrm{MN}$ delivery of antigen vaccines into the skin is to activate the adaptive immune response via antigen-presenting dendritic cells, Langerhans cells, and other cells that could prime antigen-specific immunity [16]. There has also been examples of MN devices that could effectively deliver SARS-CoV-2 spike subunit vaccines to the mice skin, such as Kim's and Kuwentrai's work [17, 18]. Encouraged by these research findings, our current study investigates the potential use of dissolvable MNs for the intradermal delivery of NP as an alternative vaccine for COVID-19. Uniquely, our MN device is composed of NP and lowmolecular weight ( $48 \mathrm{~K}$ ) hyaluronic acid (HA) that is formulated by the micromolding method [19-23]. In the mouse model, NP-MNs elicit significant B cell antibody responses and interferon-gamma- (IFN- $\gamma$-) based T cell responses compared to nonimmunized controls. Lung immunohistochemical analysis is used to examine $\mathrm{T}$ cell markers (CD4 and $\mathrm{CD} 8$ ) in $\mathrm{MN}$ vaccinated mice that might play a protective role against respiratory tract coronavirus infections [24, 25].

\section{Materials and Methods}

2.1. Nucleocapsid Protein Preparation. NP of SARS-CoV-2 (418 amino acids) was cloned and purified from E. coli as previously reported [26]. This protein was mixed at a ratio of $9: 1$ with aluminum hydroxide gel (InvivoGen).
2.2. MN Fabrication. MN patches were produced through the micromolding method. Briefly, HA (molecular weight: $48 \mathrm{~K}$, $100 \mathrm{mg} / \mathrm{mL})$ was dissolved in deionized water $(100 \mathrm{mg} / \mathrm{mL})$. NP $(25 \mu \mathrm{g})$ formulated with aluminum hydroxide gel was vortexed with the HA solution. Next, $50 \mu \mathrm{L}$ of the mixture was added to a polymethylsiloxane (PDMS) negative mold and centrifuged at 4,000 rpm for 3 minutes to make sure that all cavities in the mold were completely filled. After drying overnight at room temperature, additional HA solution was added to form the backing part of the patch. After drying, the patch was peeled off from the micromold and preserved in a dry box until use.

2.3. Animal Experiments. All BALB/c mice were obtained and purchased from the Laboratory Animal Unit of the University of Hong Kong. All animal experiments were approved by the Committee on the Use of Live Animals in Teaching \& Research, the University of Hong Kong (\#CULATR 5312-20). Vaccination was conducted using intradermal delivery of MN-formulated NP ( $25 \mu \mathrm{g} /$ mice $)$ or subcutaneous injection of NP $(25 \mu \mathrm{g} /$ mice $)$ on day 0,3 , and 7 . Blood samples were obtained from the tail vein on day 14, 21, and 28 .

2.4. IFN- $\gamma$ ELISPOT Assay. On day 28, mice were sacrificed, and spleen cells were obtained for IFN- $\gamma$ ELISPOT analysis. Briefly, $100 \mu \mathrm{L}$ of spleen cells was incubated on the IFN- $\gamma$ ELISPOT plate, which was activated for 30 minutes using $200 \mu \mathrm{L}$ DMEM media without FBS. Subsequently, $5 \mu \mathrm{g}$ of the peptide (NP and the positive inducer) was added, and cells were kept for 20 hours at $37^{\circ} \mathrm{C}$ in $5 \% \mathrm{CO}_{2}$. After the media was discarded, cells were washed using a washing buffer and incubated with the secondary biotinylated antibody for 1 hour at room temperature (RT). Finally, cells were incubated with the biotin substrate, followed by washing and drying to produce visible spots in positive wells.

2.5. Enzyme-Linked Immunosorbent Assay (ELISA). At day 14,21 , and 28 from the first vaccination, blood obtained from tail vein was centrifuged at 3,000 rpm for 30 minutes. All mice serum was stored at $-80^{\circ} \mathrm{C}$ until further characterization. A 96well ELISA plate was coated with $10 \mu \mathrm{g} / \mathrm{mL}$ of antigen (NP) in coating medium. Specifically, $100 \mu \mathrm{L}$ of the solution was added to each well at $4^{\circ} \mathrm{C}$ and kept overnight. After 12 hours, the solution was discarded, and the plate was blocked by blocking buffer (5\% milk in tris-buffered saline mixed with tween 20 [TBST]) for 3 hours at RT. The wells were then washed with TBST six times to remove milk precipitates. Next, serum collected from above was serially diluted in milk-TBST solution at the following ratios: $1: 3,1: 12,1: 48,1: 192,1: 768$, $1: 3072,1: 12288$, and $1: 49152$. The diluted serum was added to the wells and kept for 1 hour at RT. The plate was washed five times in 1X TBST and then incubated with mouse IgG, mouse IgG1, or mouse IgG2A secondary antibody diluted in milk-TBST solution at a ratio of $1: 3000$. After incubation for 1 hour at RT, the plate was washed six times using $1 \mathrm{X}$ TBST. Next, $100 \mu \mathrm{L}$ of $3,3^{\prime}, 5,5^{\prime}$-tetramethylbenzidine (TMB substrate) was added to each well and kept at $37^{\circ} \mathrm{C}$ for 30 minutes. Finally, the reaction was stopped with $\mathrm{H}_{2} \mathrm{SO}_{4}$ (50 $\mu \mathrm{L}$ per well), and the absorbance collectively was read at 


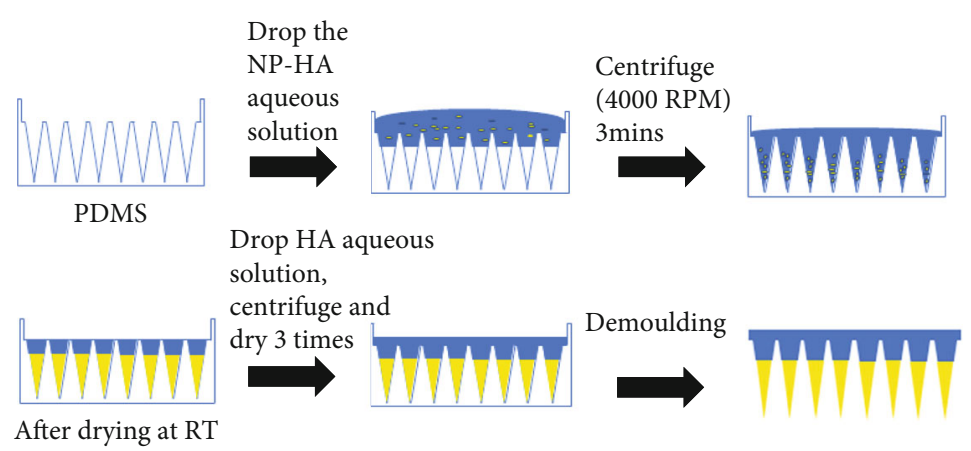

(a)

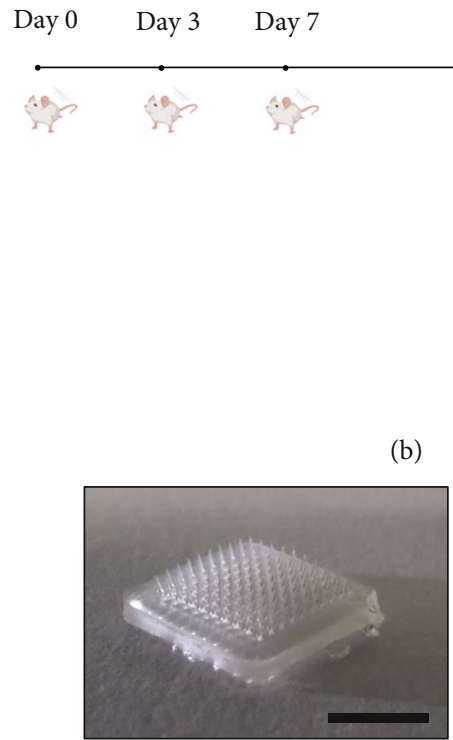

(d)

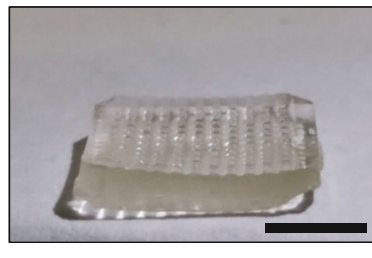

(e)

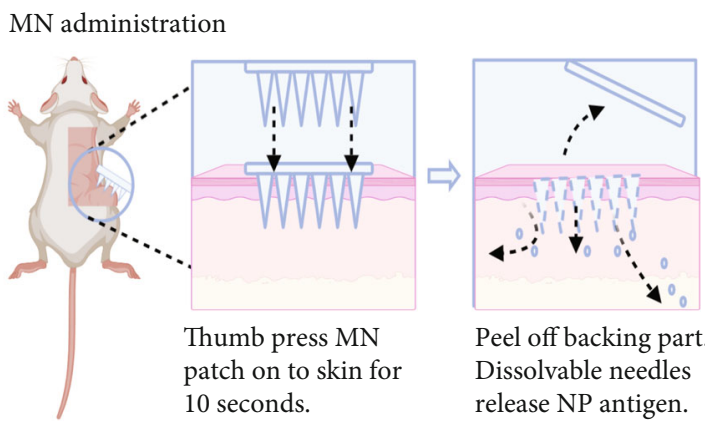

(c)

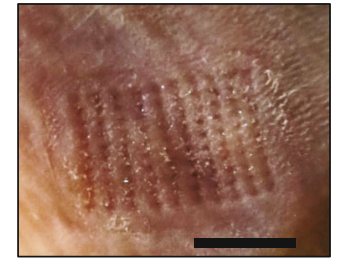

(f)

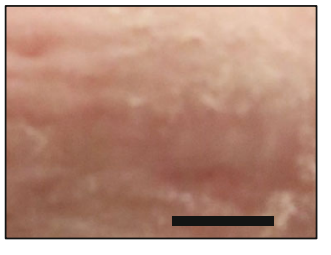

(g)

FIGURE 1: Schematic illustration of the vaccination with MN NPs: (a) MN NP fabrication process, (b) vaccination process, (c) MN NP administration on mice, image of MN NP patch (d) before deployment (tips present) (scale bar, 5 mm) and (e) after deployment (tips absent) (scale bar, $5 \mathrm{~mm}$ ), and image of the mouse skin (f) immediately after injection (scale bar, $5 \mathrm{~mm}$ ) and (g) 24 hours after injection (scale bar, $5 \mathrm{~mm}$ ).

$450 \mathrm{~nm}$. ELISA data were obtained on a Varioskan Flash spectral scanning multimode reader (Thermo Scientific).

2.6. Cryosectioning and Confocal Microscopy. After mice were sacrificed, lung tissues were harvested and fix overnight in $4 \%$ PFA at $4^{\circ} \mathrm{C}$ with gentle shaking. Tissues were washed three times for five minutes with PBS and transferred to $30 \%$ sucrose-solution ( $30 \mathrm{~g}$ Sucrose per $100 \mathrm{~mL}$ PBS). Optimal cutting temperature (OCT) compound was added in labeled cryomold and embed tissue, and cryostat sections were cut at 15-20 $\mu \mathrm{m}$ using Cryostar machine and mounted on gelatinecoated histological slides. Section slides were treated with anti-CD4 (1:50) and CD8 (1:50) mouse primary antibodies (Invitrogen) diluted in blocking buffer and incubated overnight followed by addition of Alexa488-conjugated anti-rat secondary antibodies. Sections were mounted with DAPIinfused mounting media and visualized using a fluorescence confocal microscope at $\times 20$ (LSM 800).

2.7. Statistical Analysis. All results were plotted in Prism 7 (GraphPad Software Inc., CA). Statistical comparisons between groups for ELISA were analyzed by the unpaired nonparametric $t$-test (Mann-Whitney test) using Prism 7. Statistical comparisons between groups for ELISPOT were analyzed by the unpaired parametric $t$-test using Prism 7 . For all tests, $P<0.05$ was considered statistically significant.

\section{Results}

3.1. Intradermal Delivery of NP Using Dissolvable MNs. The HA-based MN patches were made by a two-step micromolding method (Figure 1(a)) [27, 28]. Firstly, the mixture of NP and HA filled the MN tip cavities in the PDMS negative mold. Then, the backing of MNs was made using the blank HA solution. We loaded each patch $\left(1 \times 1 \mathrm{~cm}^{2}\right.$ with 100 MN tips) with $\sim 25 \mu \mathrm{g} \mathrm{NP}$, which was determined in the previous study [26]. Control patches were also made without NP. Both NP and control patches were thumb pressed into the shaved skin of BALB/c mice at days 0,3 , and $7(n=5$ per group) (Figures 1(b) and 1(c)). 10 seconds postinsertion, the MN tips detached from the base (Figures 1(d) and 1(e)). 
ELISA: day 14

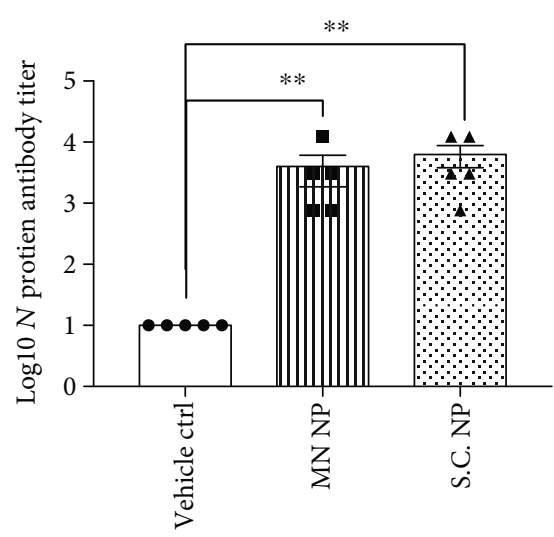

(a)

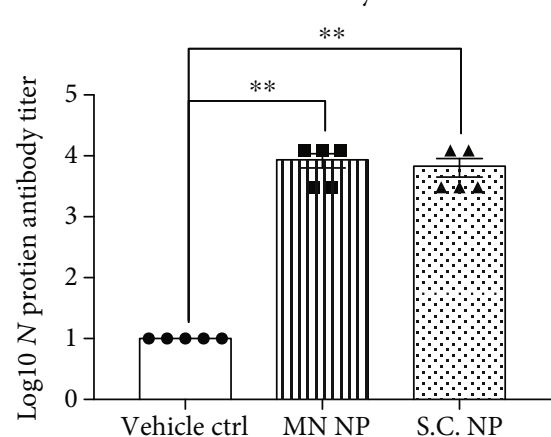

(c)

IgG2A:IgG1

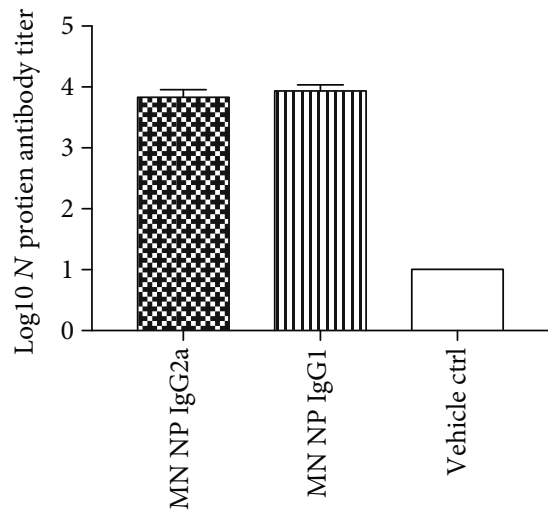

(e)
ELISA: day 21

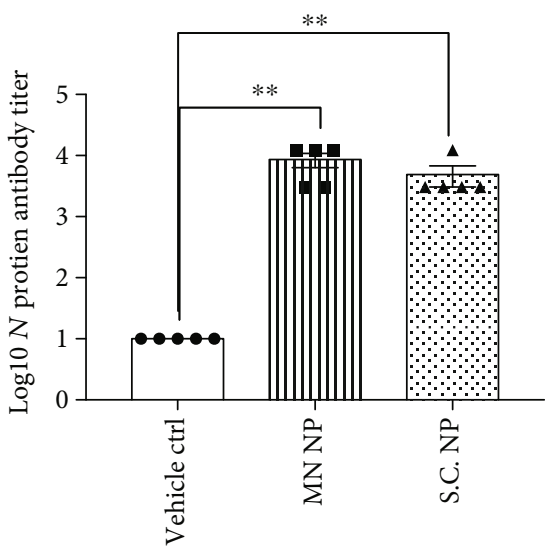

(b)

N ELISA

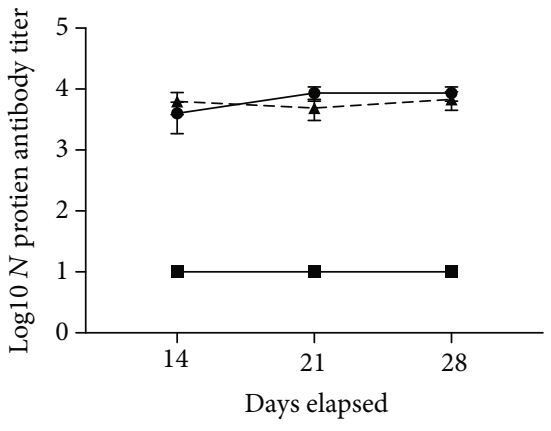

$\multimap-\mathrm{MN} \mathrm{NP}$

$\rightarrow$ Vehicle ctrl

- - S.C. NP

(d)

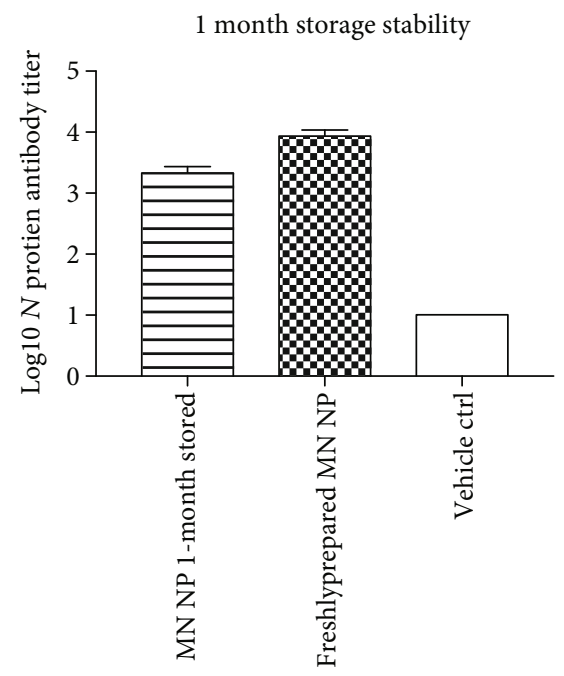

(f)

Figure 2: Specific B cell antibody responses to vaccination. ELISA results of serum at (a) day 14, (b) day 21, and (c) day 28 postvaccination, showing $\log 10$ antibody titers against the $\mathrm{N}$ protein in the S.C. NP immunization group, MN NP immunization group, and vehicle-control group. (d) Summary of $\log 10$ mean NP antibody titers from S.C. NP, MN NP, and vehicle-control MN groups. (e) ELISA results of serum at day 28 postvaccination, showing $\log 10 \mathrm{IgG} 1$ and IgG2A antibody titers against the N protein in the MN NP immunization group. (f) ELISA results of mice serum at day 28 postvaccination, showing $\log 10$ antibody titers against NP in the freshly prepared MN NP immunization group, 1 month stored MN NP immunization group and vehicle-control group. ELISA absorbance measurements at $450 \mathrm{~nm}$ were normalized to standard cut-off values. Student's unpaired nonparametric $t$-test (Mann-Whitney) was used with multiple $t$-test adjustment. Data were expressed as mean \pm SEM. ${ }^{*} P<0.05,{ }^{* *} P<0.01,{ }^{* * *} P<0.001,{ }^{* * * *} P<0.0001$, ns refers to "not significant". 


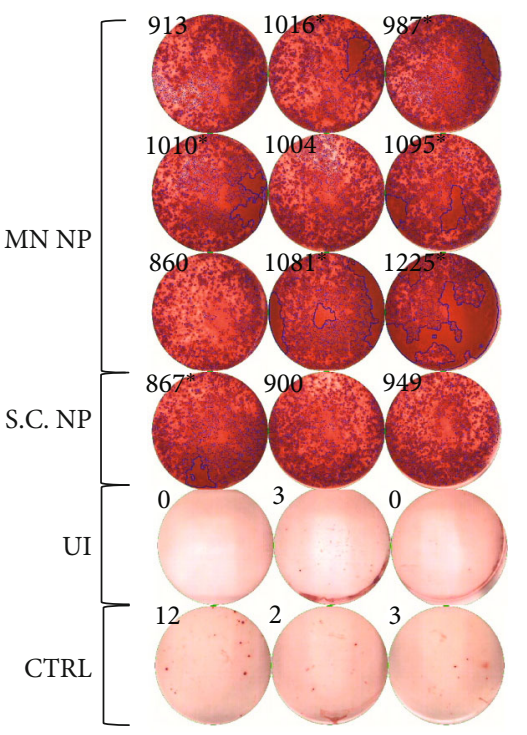

(a)

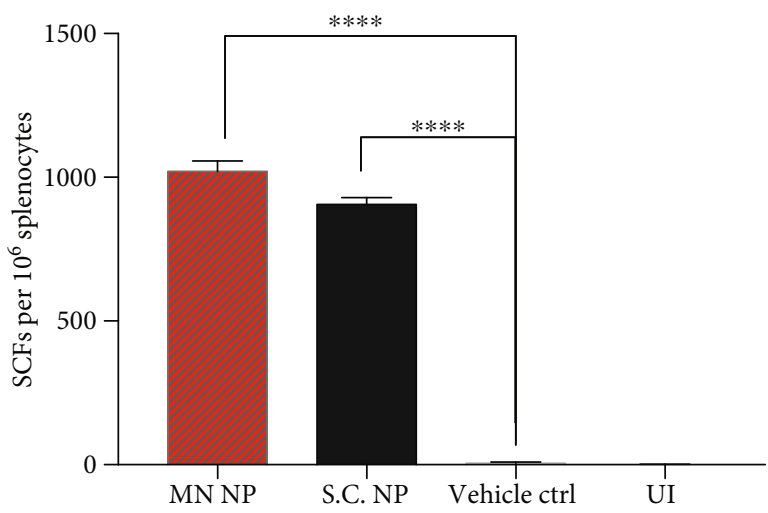

(b)

FIgURE 3: IFN- $\gamma$ T cell responses. (a) IFN- $\gamma$ ELISPOT plate counts for S.C. NP stimulated, MN NP stimulated, blank MN stimulated (CTRL), and nonstimulated (UI) groups. (b) Bar chart shows mean IFN- $\gamma$ ELISPOT counts for all four groups. Student's unpaired parametric $t$-test was used. Data were expressed as mean $\pm \mathrm{SD} .{ }^{*} P<0.05,{ }^{* *} P<0.01,{ }^{* * *} P<0.001,{ }^{* * * *} P<0.0001$, ns refers to "not significant".
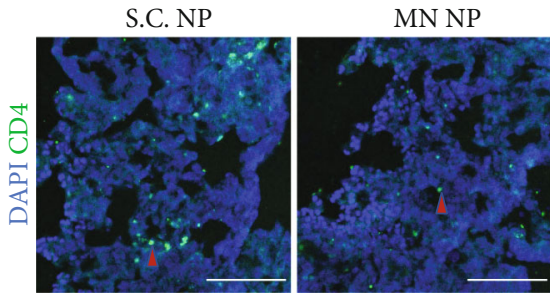

(a)
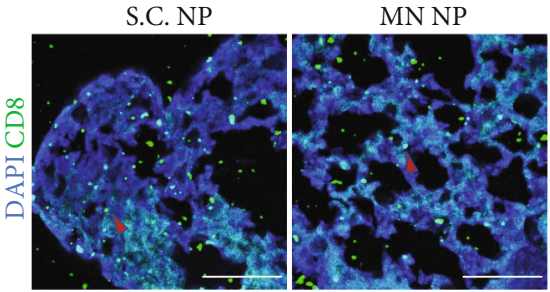

(b)

Figure 4: Immunohistochemical staining of lung tissues from MN-based NP immunized mice. At day 29, mice from the S.C. NP immunization group, MN NP immunization group, and vehicle control groups were sacrificed and lung tissues were collected and subjected to IHC staining of CD4 (a) and CD8 (b) markers. Sections were viewed under LSM800 confocal microscope. Scale bars, $100 \mu \mathrm{m}$.

There were MN marks right after the application, which disappeared after 24 hours (Figures $1(\mathrm{f})$ and $1(\mathrm{~g})$ ).

\subsection{MN-Delivered NP Induced Specific B Cell Antibody} Responses. The specific $B$ cell antibody response post the delivery of NP was studied through the ELISA assay of IgG antibodies in sera against SARS-CoV-2. There were three groups including subcutaneously injected (S.C.) NP $(25 \mu \mathrm{g} /$ mice), MN-delivered NP (25 $\mu \mathrm{g} /$ mice), or MN-delivered vehicle controls. There was no significant difference between the S.C. NP group and the MN NP group (Figures 2(a)-2(d)). Both groups showed strong immune response. NP antibody titers could be seen at day 14 after the first immunization, which elevated at day 21 , and further increased to a maximum antibody titer of $1.2 \times 10^{4}$ at day 28 . By day 28 , an average antibody titer of over $8.6 \times 10^{3}$ was detected, indicating that both the MN and S.C. delivery of NP were highly effective. In comparison, there was no comparable immune response in the control group. Importantly, the MN NP group exhibited a balanced ratio of IgG1 and IgG2A NP-specific antibodies, which is vital for viral clear- ance (Figure 2(e)). Furthermore, MN NP patches that were stored in a dehumidifier (25 degrees) induced comparable NP-specific antibodies in mice compared to freshly prepared MN NP patches at day 28 after immunization (Figure 2(f)).

3.3. MN-Delivered NP Elicited Specific T Cell Responses. We investigated the T cell IFN- $\gamma$ response in mouse splenocyte immunization by S.C. NP, MN NP, and vehicle-control MN by using ELISPOT with the NP protein $(5 \mu \mathrm{g} / \mathrm{mL})$ as the stimulator. Ionomycin was used as the positive inducer, and FBSfree medium was used as the negative inducer. As shown in Figures $3(\mathrm{a})$ and $3(\mathrm{~b})$, significant IFN- $\gamma$ was released by T cells by day 29 in the S.C. NP and MN NP groups compared to vehicle and noninduced controls. The elevated levels of IFN$\gamma$ producing $\mathrm{T}$ cells may produce strong antiviral protection against SARS-CoV-2.

3.4. Immunohistochemical Staining of T Cell Markers within Mouse Lung Tissues Posts the Stimulation with MNDelivered NP. Zhao et al. showed that Venezuelan equine 
encephalitis replicons (VRP) SARS-CoV NP induced CD4+ $\mathrm{T}$ cells in the lungs, which played an important role in SARS-CoV and MERS-CoV protection [24]. Habel et al. also showed that CD8+ T cell responses in the lungs were critical for COVID-19 recovery [25]. Based on these observations, we examine the presence of CD4 and CD8 T cell markers in the mouse lungs post the delivery of NPs (Figure 4). CD4 staining represents $\mathrm{T}$ helper cells, whereas CD8 represents cytotoxic T cells. Both types of immune cells in the lungs are present after the $\mathrm{MN}$ and S.C. delivery of NP, which may confer protection towards SARS-CoV-2.

\section{Discussion and Conclusion}

This study reports the successful intradermal delivery of NP using dissolvable HA MNs that could induce specific B cell antibodies and IFN- $\gamma$ T cell responses in mice. This MN formulation is composed of NP antigens, HA, and aluminum hydroxide gel adjuvant.

Previously, Kim et al. delivered recombinant coronavirus vaccines (SARS-CoV-2-S1 and SARS-CoV-2-S1fRS09 subunit) using carboxymethyl cellulose-based $\mathrm{MN}$ delivery platforms [17]. Moreover, our team previously explored the use of HA-based MN delivery platform to deliver S-RBD (SARS-CoV-2-S1) in mice and obtained similar results [18]. In this work, we investigated the use of HA-based MN delivery platform to deliver NP in mice. The rationale behind this is to illustrate the immunogenicity of $\mathrm{NP}$ as an alternative antigen that could induce specific $\mathrm{B}$ and $\mathrm{T}$ cell responses in a similar manner to the conventional spike protein SARS$\mathrm{CoV}-2$ vaccine target.

Our work supports the NP as an alternative vaccine candidate for SARS-CoV-2. We showed that MN NP could trigger significant $\mathrm{B}$ cell antibody responses and significant $\mathrm{T}$ cell IFN- $\gamma$ responses. Notably, T cell IFN- $\gamma$ responses towards viral antigens have been reported as critical antiviral protective factors that partake in the eradication of COVID19 [26]. T cell responses may be as vital as the induction of specific B cell antibodies against SARS-CoV-2 $[29,30]$. Previous work by Zhao et al. showed that CD4+ T cells in the lungs are induced after intranasal immunization by VRP SARS$\mathrm{CoV}$ nucleocapsid protein, and that these cells play an important role in SARS-CoV and MERS-CoV protection [24]. Habel et al. also recently discovered that CD8+ T cell immunity is vital for virus protection amongst COVID-19 patients. Thus, we performed immunohistochemical staining of lung tissues for MN NP, S.C. NP, and control MN groups to demonstrate presence of CD4 and CD8 T cell markers in the lungs of NP immunized mice. On the other hand, Wang and colleagues discovered that the neutralization activity of spike-targeting mRNA vaccines decreased against the mutated COVID-19 strain, 501Y.V2 [31]. Furthermore, a recent study by Li et al. demonstrated the effects of different mutations on the spike that could hinder the effects of neutralizing antibodies against the antigen. For instance, the N234Q mutation decreased neutralization sensitivity against different monoclonal antibodies. Whether these mutations have a direct effect towards protective vaccine efficacy remains to be investigated [32]. These findings support the development of additional nonspike candidates, such as NP, to fully eradicate new mutated strains. On the other hand, there are emerging clinical studies about combining different treatments. For instance, Hung et al. administered either a 14-day combination of lopinavir $400 \mathrm{mg}$ and ritonavir $100 \mathrm{mg}$ every $12 \mathrm{~h}$, ribavirin $400 \mathrm{mg}$ every $12 \mathrm{~h}$, and three doses of 8 million international units of interferon beta- $1 \mathrm{~b}$ on alternate days (combination group) or to 14 days of lopinavir $400 \mathrm{mg}$ and ritonavir $100 \mathrm{mg}$ every $12 \mathrm{~h}$ (control group) to randomly assigned COVID-19 patients in a phase 2 trial [33]. Therefore, another consideration is to combine NP vaccines with spike vaccines to augment efficacy. Interestingly, we also discovered that it is possible to combine 2 proteins into a single microneedle (supplementary Figure S1).

The MN-based intradermal delivery is a unique method for administering vaccines with distinct benefits such as noninvasiveness, full biocompatibility, fast vaccination deployment, sustained drug release, ease of use, and reduction of logistic costs and wastage. In our work, we used dissolvable HA MNs to administer NP vaccines, allowing sustained drug release into tissue layers beneath the skin for triggering adaptive immunity. The sustained release of NP vaccines increases drug utility by reducing fluctuations in steady-state drug levels and enhances the safety margin of the drug activity [34]. Microneedle skin patches (area per patch $=10 \mathrm{~mm} \times 10 \mathrm{~mm}$, individual needle length $=1 \mathrm{~mm}$ ) may be administered quickly by thumb pressing on to the skin for at least 10 seconds before peeling. The fast and straight-forward administration process of MN devices dominates over conventional S.C. procedures that require trained personnel to accurately inject the vaccine underneath the skin. As mentioned in our manuscript, microneedle penetration of the mice skin led to penetration marks indicating successful delivery of microneedle components (Figure 1(f)). We found that these penetration marks disappeared overnight after administration (Figure 1(g)). There was lack of scarring on the mice skin which also supports the noninvasive nature of MN vaccine delivery. Our MN devices are formulated using biodegradable $50 \mathrm{kDa}$ hyaluronic acid (HA) which is a wellstudied substance known for its natural occurrence in the skin, good solubility, and lack of reported side effects. The biodegradable nature of $\mathrm{MN}$ vaccines prevents the requirement for safe needle disposal and waste, unlike for S.C. injections. Furthermore, preformulated $\mathrm{MN}$ vaccines can be stored at room temperature which promotes vaccine storage and distribution in developing countries with limited cold chain. Alternatively, several factors may limit the efficiency of the MN delivery system for NP, such as possible loss of antigen activity during the $\mathrm{MN}$ formulation and storage. However, we found that the titers of specific NP antibodies produced by the MN method were comparable to the S.C. injection method (Figure 2). The NP-specific IgG2A and IgG1 titers induced by the MN NP group were also comparable, indicating a balanced Th2 and Th1 immunity. Additionally, 1 month's storage of MN NP in a dehumidifier does not seem to affect the activity of inducing NP specific antibodies in mice compared to freshly prepared patches (Figure 2).

In the next developmental stage, the clinical translation of $\mathrm{MN}$ vaccine technology still requires the optimization several other key factors. These factors include ensuring device sterility within germ-free production laboratory sites, 
promoting cost-effective fabrication in large scale, and developing applicators for consistent deployment of MNs. We hope to address these factors in our future work to optimize the HA MN delivery for clinical testing of human COVID-19 patients. For our future work, we also plan to conduct further clinical investigations on the dosing regimen required for combining MN NP with MN S-RBD as a combination therapy regimen for COVID-19 patients.

\section{Data Availability}

The data that support the findings of this study are available from the corresponding author upon request.

\section{Conflicts of Interest}

The authors declare that they have no conflicts of interest.

\section{Authors' Contributions}

Chaiyaporn Kuwentrai and Jinming Yu contributed equally to this work.

\section{Acknowledgments}

This work was supported by the Health and Medical Research Fund (COVID190117), the Shenzhen Peacock Team Project (KQTD2015033117210153), Shenzhen Science and Technology Innovation Committee Basic Science Research Grant (JCYJ20170413154523577), and China Postdoctoral Science Foundation (2019 M663167) to JDH. C.X. acknowledges the funding support from the City University of Hong Kong (\#9610472), General Research Fund (GRF) of University Grant Committee of Hong Kong (UGC) Research Grant Council (RGC) (\#9042951), and NSFC/RGC Joint Research Scheme (N_CityU118/20). We would like to acknowledge BioRender for providing an illustrative platform for producing figures. JDH is supported by the L \& T Charitable Foundation.

\section{Supplementary Materials}

We have included one additional supplementary file containing the graphical abstract and Figure S1. (Supplementary Materials)

\section{References}

[1] M. Regueiro, B. G. Feagan, B. Zou et al., "The COVID-19 vaccine development landscape," Nature Reviews. Drug Discovery, vol. 19, no. 5, pp. 305-306, 2020.

[2] J. F.-W. Chan, S. Yuan, K. H. Kok et al., “A familial cluster of pneumonia associated with the 2019 novel coronavirus indicating person-to-person transmission: a study of a family cluster," The Lancet, vol. 395, no. 10223, pp. 514-523, 2020.

[3] World Health Organization (WHO), DRAFT landscape of COVID-19 candidate vaccines, World Health Organization, 2020, https://www.who.int/docs/default-source/a-future-forchildren/novel-coronavirus_landscape_covid-19.pdf?sfvrsn= 4d8bd201_1.
[4] N. K. Dutta, K. Mazumdar, and J. T. Gordy, "The nucleocapsid protein of SARS-CoV-2: a target for vaccine development," Journal of Virology, vol. 94, no. 13, 2020.

[5] R. McBride, M. van Zyl, and B. Fielding, "The coronavirus nucleocapsid is a multifunctional protein," Viruses, vol. 6, no. 8, pp. 2991-3018, 2014.

[6] H. Z. Chen, L. L. Tang, X. L. Yu, J. Zhou, Y. F. Chang, and $\mathrm{X}$. Wu, "Bioinformatics analysis of epitope-based vaccine design against the novel SARS-CoV-2," Infectious Diseases of Poverty, vol. 9, no. 1, p. 88, 2020.

[7] W. Zeng, G. Liu, H. Ma et al., "Biochemical characterization of SARS-CoV-2 nucleocapsid protein," Biochemical and Biophysical Research Communications, vol. 527, no. 3, pp. 618-623, 2020.

[8] O. S. Chukwudozie, R. C. Chukwuanukwu, O. O. Iroanya et al., "Attenuated subcomponent vaccine design targeting the SARS-CoV-2 nucleocapsid phosphoprotein RNA binding domain: in silico analysis," Journal of Immunology Research, vol. 2020, 2020.

[9] Y. Peng, A. J. Mentzer, G. Liu et al., "Broad and strong memory CD4(+)and CD8(+)T cells induced by SARS-CoV-2 in UK convalescent individuals following COVID-19," Nature Immunology, vol. 16, pp. 1336-1345, 2020.

[10] R. Zhou, T. KK, Y. C. Wong et al., "Acute SARS-CoV-2 infection impairs dDendritic cell and T cell responses," Immunity, vol. 53, pp. 864-877.e865, 2020.

[11] L. A. Jackson, E. J. Anderson, N. G. Rouphael et al., "An mRNA vaccine against SARS-CoV-2 - preliminary report," New England Journal of Medicine, vol. 383, no. 20, pp. 19201931, 2020.

[12] E. Weir and K. Hatch, "Preventing cold chain failure: vaccine storage and handling," CMAJ, vol. 171, no. 9, p. 1050, 2004.

[13] C. I. Shin, S. D. Jeong, N. S. Rejinold, and Y. C. Kim, "Microneedles for vaccine delivery: challenges and future perspectives," Therapeutic Delivery, vol. 8, no. 6, pp. 447-460, 2017.

[14] M. Dicko, A. Q. Oni, S. Ganivet, S. Kone, L. Pierre, and B. Jacquet, "Safety of immunization injections in Africa: not simply a problem of logistics," Bulletin of the World Health Organization, vol. 78, no. 2, pp. 163-169, 2000.

[15] E. L. Giudice and J. D. Campbell, "Needle-free vaccine delivery," Advanced Drug Delivery Reviews, vol. 58, no. 1, pp. 6889, 2006.

[16] N. Li, N. Wang, X. Wang, Y. Zhen, and T. Wang, "Microneedle arrays delivery of the conventional vaccines based on nonvirulent viruses," Drug Delivery, vol. 23, no. 9, pp. 3234-3247, 2016.

[17] E. Kim, G. Erdos, S. Huang et al., "Microneedle array delivered recombinant coronavirus vaccines: immunogenicity and rapid translational development," eBioMedicine, vol. 55, p. 102743, 2020.

[18] C. Kuwentrai, J. Yu, L. Rong et al., "Intradermal delivery of Receptor-Binding Domain of SARS-CoV-2 spike protein with dissolvable microneedles to induce humoral and cellular responses in mice," Bioengineering \& translational medicine, vol. 6, no. 1, 2020 https://aiche.onlinelibrary.wiley.com/doi/ full/10.1002/btm2.10202.

[19] E. Larrañeta, M. Henry, N. J. Irwin, J. Trotter, A. A. Perminova, and R. F. Donnelly, "Synthesis and characterization of hyaluronic acid hydrogels crosslinked using a solvent-free process for potential biomedical applications," Carbohydrate polymers, vol. 181, pp. 1194-1205, 2018. 
[20] T. Waghule, G. Singhvi, S. K. Dubey et al., "Microneedles: a smart approach and increasing potential for transdermal drug delivery system," Biomedicine \& Pharmacotherapy, vol. 109, pp. 1249-1258, 2019.

[21] W. Martanto, S. P. Davis, N. R. Holiday, J. Wang, H. S. Gill, and M. R. Prausnitz, "Transdermal delivery of insulin using microneedles in vivo," Pharmaceutical Research, vol. 21, no. 6, pp. 947-952, 2004.

[22] S. H. Bariya, M. C. Gohel, T. A. Mehta, and O. P. Sharma, "Microneedles: an emerging transdermal drug delivery system," The Journal of Pharmacy and Pharmacology, vol. 64, no. 1, pp. 11-29, 2012.

[23] B. Xu, W. K. Raja, and S. Lee, "Silk-based microneedle transdermal immunization with EV71 vaccines elicits potent immune responses in mice," Journal of Vaccines \& Immunization, vol. 4, pp. 1-8, 2016.

[24] J. Zhao, J. Zhao, A. K. Mangalam et al., “Airway memory CD4 $+\mathrm{T}$ cells mediate protective immunity against emerging respiratory coronaviruses," Immunity, vol. 44, no. 6, pp. 1379-1391, 2016.

[25] J. R. Habel, T. H. O. Nguyen, C. E. van de Sandt et al., "Suboptimal SARS-CoV-2-specific CD8 ${ }^{+} \mathrm{T}$ cell response associated with the prominent HLA-A $*$ 02:01 phenotype," Proceedings of the National Academy of Sciences, vol. 117, no. 39, pp. 24384-24391, 2020.

[26] B. Z. Zhang, Y. F. Hu, L. L. Chen et al., "Mining of epitopes on spike protein of SARS-CoV-2 from COVID-19 patients," Cell Research, vol. 30, no. 8, pp. 702-704, 2020.

[27] M. Zheng, Z. Wang, H. Chang et al., "Osmosis-powered hydrogel microneedles for microliters of skin interstitial fluid extraction within minutes," Advanced Healthcare Materials, vol. 9, no. 10, p. 1901683, 2020.

[28] H. Chang, M. Zheng, S. W. T. Chew, and C. Xu, "Advances in the formulations of microneedles for manifold biomedical applications," Advanced Materials Technologies, vol. 5, no. 4, p. 1900552, 2020.

[29] A. Grifoni, D. Weiskopf, S. I. Ramirez et al., "Targets of T cell responses to SARS-CoV-2 coronavirus in humans with COVID-19 disease and unexposed individuals," Cell, vol. 181, no. 7, pp. 1489-1501.e15, 2020.

[30] Q. X. Long, X. J. Tang, Q. L. Shi et al., "Clinical and immunological assessment of asymptomatic SARS-CoV-2 infections," Nature Medicine, vol. 26, no. 8, pp. 1200-1204, 2020.

[31] Y. Wang, "In the face of the more fearsome variant of South Africa, top scientists are rapidly making mRNA vaccination effectiveness validation; Suggest key responses," Hanson Clinical Researchhttps://xw.qianzhan.com/analyst/detail/329/ 210121-67ae5e45.html.

[32] Q. Li, J. Wu, J. Nie et al., "The Impact of mutations in SARSCoV-2 spike on viral infectivity and antigenicity," Cell, vol. 182, no. 5, pp. 1284-1294.e9, 2020.

[33] I. F. N. Hung, K. C. Lung, E. Y. K. Tso et al., “Triple combination of interferon beta-1b, lopinavir-ritonavir, and ribavirin in the treatment of patients admitted to hospital with COVID-19: an open-label, randomised, phase 2 trial," The Lancet, vol. 395, no. 10238, pp. 1695-1704, 2020.

[34] K. M. Kwon, S. M. Lim, S. Choi et al., "Microneedles: quick and easy delivery methods of vaccines," Clinical and Experimental Vaccine Research, vol. 6, no. 2, pp. 156-159, 2017. 